A two-stage modelling approach for the analysis of the stress distribution in anchorage zones of pre-tensioned, concrete elements

Peer-reviewed author version

STEENSELS, Rik; Vandewalle, Lucie; VANDOREN, Bram \& DEGEE, Herve (2017) A two-stage modelling approach for the analysis of the stress distribution in anchorage zones of pre-tensioned, concrete elements. In: ENGINEERING STRUCTURES, 143, p. 384-397.

DOI: $10.1016 /$ j.engstruct.2017.04.011

Handle: http://hdl.handle.net/1942/23670 


\title{
A two-stage modelling approach for the analysis of the stress distribution in anchorage zones of pre-tensioned, concrete elements
}

\author{
R. Steensels ${ }^{\mathrm{a}, *}$, L. Vandewalle ${ }^{\mathrm{b}}$, B. Vandoren ${ }^{\mathrm{a}}$, H. Degée ${ }^{\mathrm{a}}$ \\ ${ }^{a}$ Faculty of Engineering Technology, Agoralaan Gebouw H, 3590 Diepenbeek Belgium \\ ${ }^{b}$ Department Civil Engineering, Kasteelpark Arenberg 40 bus 2448, 3001 Leuven Belgium
}

\begin{abstract}
An innovative strategy for the analysis and design of the anchorage zones of pre-tensioned, concrete girders is presented. In this approach, the bond behaviour of the prestress strands is first characterised in a small-sized beam model. A new relation between the slip and radial strain of the prestress strand is introduced and used together with the radial stress-strain relation resulting from a thick-walled cylinder model to establish the bond-slip behaviour at the steel-concrete interface. This bond behaviour is implemented in a numerical model and validated via a comparison of the computed transfer length with the results of two experimental campaigns. Next, the bond-slip relation of the small-scale model is applied in full-scale models of pre-tensioned, concrete girders to derive the stress distribution in the anchorage zones. The non-linear material behaviour of concrete is taken into account and a comparison of the numerical results with full-scale experimental data is made. An acceptable agreement is achieved between the experimental results and the numerical calculations regarding the bond behaviour and transfer lengths as well as the crack patterns and the stress values in the reinforcement bars. This efficient modelling approach allows for a full analysis of the anchorage zone based solely on the geometrical and material properties known at the design stage.
\end{abstract}

Keywords: Pre-tensioned concrete, Prestressed concrete, Transfer length, Bond behaviour, Two-stage analysis

\section{Introduction}

Although the use of prestressed concrete girders for structures involving large spans is widespread nowadays, field observations show that some of these elements still exhibit non negligible cracking in the anchorage zone due to a combination of large prestress forces and inadequate anchorage zone reinforcement design. The transfer of the prestress force from the prestress tendon to the surrounding reinforcement and the spreading of these forces over the height of the element cause a non-linear stress distribution in the anchorage zone which is not easily controlled. Furthermore, the commonly used EulerBernouilli beam theory is not suited to describe the stress field in this zone since the cross-section of the element under prestress load does not remain plane and does not stay perpendicular to the neutral axis. Therefore, characterising the stress distribution in the anchorage zones is often tackled by resorting to specifically derived analytical or numerical approaches, often involving simplifications.

Advanced numerical models, validated against empirical data, have recently been applied in order to get a better understanding of the behaviour of these zones in view of a more efficient and economic design of anchorage zone reinforcement. Tuan et al. [1] studied analytical models of the anchorage zone using conventional methods like the strut-and-tie method and Gergely-Sozen method but also more advanced numerical finite

\footnotetext{
${ }^{*}$ Corresponding author

Email address: rik.steensels@uhasselt.be (R. Steensels)
}

element models. Okumus [2, 3, 4] also used the finite element method to analyse the stress distribution of the end zones of pre-tensioned elements but included non-linear concrete material behaviour. A study of the tensile stresses in the anchorage zones of four full-scale beams was conducted by O'Callaghan [5] who investigated the tensile stresses in the anchorage zone empirically. Resulting from these researches, the value of the prestress force, the eccentricity at which it is applied and the value of the transfer length are identified as being the main parameters governing the cracking behaviour in the end zones. Furthermore, an insight is provided into the cause of anchorage zone cracks and suggestions concerning the anchorage zone reinforcement lay-out are made.

As just stated, proper evaluation of the tensile stresses in the anchorage zone and a consequent effective design of the corresponding reinforcement lay-out in this zone require a correct assessment of the transfer length. This transfer length is the length, measured from the extremity of the member, necessary to lead to the the full transfer of the prestressing force from the tendon to the surrounding concrete [6]. A short transfer length will induce high tensile stresses within the anchorage zone and may thus lead to cracks which may deteriorate the structural integrity of the element. The transfer length is essentially governed by the bond behaviour at the steel concrete interface and the value of the bond strength depends on a range of parameters. Extensive experimental research has been performed in order to identify and investigate these influences. It is found that concrete confinement is one of the most important aspects governing the bond behaviour, as evidenced by the experimental 
campaigns of Galvez et al. [7], Marti-Vargas et al. [8], Benitez et al. [9] and Torre-Cassanova et al. [10]. This concept of concrete confinement incorporates geometrical parameters, such as concrete cover and strand diameter, and material parameters, such as the concrete strength.

The mechanical action of the strand also affects the bond behaviour. Gustavson [11] and Moon et al. [12] notice a significant contribution of the indentations and helical shape of the strand to the bond strength. The experimental campaign of Marti-Vargas [13] also showed that other factors like the water/cement ratio of the concrete mixture influences the value of the transfer length. Moreover, this influence is higher for concrete mixtures with a high cement content. However, most formulae for the evaluation of the transfer length available in current codes and standards are still based on empirical formulations and only take into account a limited part of the numerous influencing parameters [14, 15, 16, 17]. Furthermore, Royce et al. [18] and Marti-Vargas et al. [8] showed that these different normative formulae are providing a substantial range of values for the transfer length and thus lack accuracy.

Modelling the transfer of the prestress force in pre-tensioned, concrete elements has also been investigated extensively over the past decade. Ayoub and Fillippou [19] used a bond-slip relation similar to the one that can be found in the CEB Fib Model code 2010 [17]. Some of the parameters applied in this bond model were evaluated beforehand by Tabatabai and Dickson [20] through an experimental campaign.

Lundgren [21] advocated that the bond behaviour should not be inserted into the model as a fixed input but that it should result rather from an analysis. Galvez et al. [7, 22] simulated the bond behaviour by implementing a non-associative yield function representing the relation between the bond strength and the compressive stress at the steel-concrete interface. The confinement of the surrounding concrete was taken into account in the model using a cohesive crack model. The confinement effect can alternatively be taken into consideration using a thickwalled cylinder model. This approach is based on the work of Timoshenko [23] and describes the constitutive behaviour of the concrete surrounding a prestressing strand based on the geometric and material parameters of the specimen. The resulting stress-strain relation is then used as an input for determining the bond-slip relation. This way of assessing the bond-behaviour and the confinement of the surrounding concrete was applied by Den Uijl [24], Fellinger [25], Oh et al. [26] and Benitez et al. [27].

Very recently, Abdelatif et al. [28] presented a comparison of three different models of the prestress transfer. The first model was an analytical model using a thick-walled cylinder approach. The two other models consisted of a 2D axi-symmetric finite element model with linear elastic material behaviour and of a full 3D finite element model with non-linear concrete constitutive behaviour. From this research, an analytical formulation of the stress distribution along the transfer length is proposed. Furthermore, it is concluded from the non-linear finite element model that the thick-walled cylinder concept is justified to describe the confinement of the prestress tendon.

Arab et al. [29, 30] compared several modelling techniques concerning the prestress tendons. One model uses an extrusion technique, a second model is built following the concept of embedded reinforcement in which the prestress tendons are implemented using one-dimensional elements which are embedded in the concrete continuum elements. For both models, bond-slip behaviour is achieved by implementing the frictional nature at the concrete-strand interface. However, in the extruded model, this is achieved through contact surface algorithms while the embedded model uses nodal constraints and master-slave connections It is concluded that a correct assessment of the overall behaviour of the pre-tensioned elements can be achieved with both techniques [29]. However, the local behaviour surrounding the strand was better approximated with the extrusion technique even though this latter approach was numerically more expensive. To allow for the correct evaluation of the self-weight of the elements, the prestress bed was also modelled together with contact formulations. This time the frictional behaviour was minimised in order to limit the influence on the modelled girder in the axial direction. In the vertical direction, hard contact behaviour is implemented which prevents the girder from merging with the prestress bed but allows for the uplift from the casting bed.

As a conclusion of the review of available references dealing with the transfer of the prestress force and with the bond behaviour in pre-tensioned elements, it can be stated that focus is mostly set on the modelling of the bond-slip behaviour at a local scale, evaluating the transfer length and its influences for small beams with one strand.

In this general context, the present paper proposes an efficient modelling strategy aiming at characterising the non-linear stress distribution within the anchorage zone of pre-tensioned girders on the base of the limited number of geometric and material parameters that can be identified at the design stage. This is achieved by the application of a decoupled two-stage analysis. In the first stage, the bond-slip behaviour is evaluated by modelling a small-sized prestressed beam with only one strand using equivalent strand confinement and material properties to those of the full-scale girder. The equivalent strand confinement is achieved by evaluating an effective concrete cover using the actual geometric values of the concrete cover and of the clear spacing between the prestress strands of the full-scale model. This effective cover is then used in the small-scale model to compute the bond-slip behaviour at the steel strand interface. As a result, the appropriate bond-slip behaviour and transfer lengths of the strand can be determined through the use of a simple and numerically affordable model. In the second stage, the computed bond-slip behaviour is implemented into a fullscale model with non-linear material behaviour which in turn allows for the correct portrayal of the stress state in the anchorage zone. This includes the stress behaviour of the reinforcing steel as well as the concrete material, including crack prediction if relevant. The proposed approach is compared with experimental results in order to validate the prediction of the transfer length and of the bond-slip behaviour of the pre-tensioned strands. The appropriate strain distribution in the anchorage zone is also checked by referring to existing experimental data. 


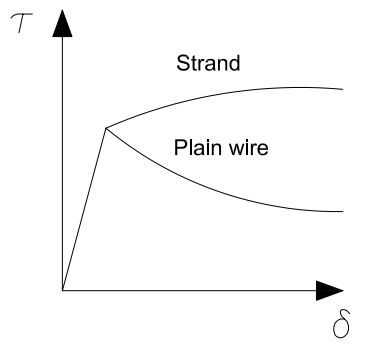

Figure 1: Bond-slip relation of 7-wire strand versus plain wire

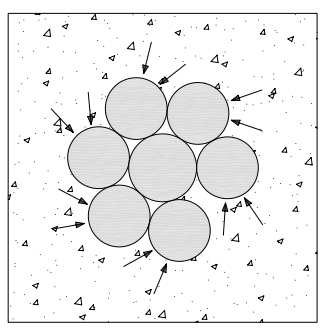

(a) Pitch effect

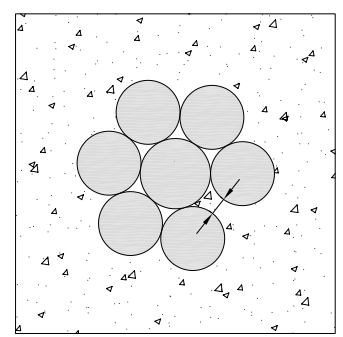

(b) 'Lack-of-fit'
Figure 2: Mechanical resistance phenomena

\section{Modelling bond behaviour}

\subsection{Bond mechanism of 7-wire strands}

The bond behaviour of 7-wire strands differs quite significantly from that of plain wires, as can be concluded from experimentally identified bond-slip relations [31] (figure 1). The bond strength at the steel-concrete interface is attributed to three aspects; chemical adhesion, mechanical resistance, and frictional forces between steel and concrete [32]. The chemical adhesion at the steel-concrete interface is very small and can be neglected [21]. Mechanical resistance of the strand itself is of great importance when ribbed or indented bars are concerned $[9,11,24]$. The mechanical resistance can be converted into an equivalent frictional behaviour which depends on the characteristics of the tendon and of the surrounding concrete [22, 24]. Two major phenomena can be denoted concerning the mechanical resistance, the pitch effect (figure 2a) and the 'lack-of-fit' effect (figure 2b). When a prestress strand is de-tensioned, it will rotate while slipping into the concrete due to the fact that the outer wires of a 7-wire strand are twisted around the middle wire. The tendon tries to follow the helical grooves during de-tensioning and is thus inclined to a change of pitch. However, this pitch change is restrained by the surrounding concrete and creates a torsional moment which is balanced by compressive stresses at the steel-concrete interface, improving the bond characteristics of the strand. The frictional aspect of the bond behaviour is conditioned by the radial compressive stresses at the steel-concrete interface and depends on the level of confinement created by the surrounding concrete [24].

A further increase of the bond strength could theoretically result from the 'lack-of-fit' effect mentioned above. This phenomenon refers actually to the lack of fit of the outer wires around the middle wire of a 7-wire strand creating a gap between the outer wires. This gap can be filled with cementitious material, causing additional restraint of the pitch change and thus increasing the contact stresses around the perimeter of the strand. Some authors like Stocker and Sozen [33] investigated the importance of these effects on the basis of experimental pull-out tests. No substantial change of pitch was noticed during these tests and it was concluded that the influence of this effect was marginal due to the low torsional stiffness of the strand. However, the test set-up used by Stocker and Sozen was questioned by Russel and Burns [34] who state that the boundary conditions of the original tests were unsatisfactory and minimised the effect of the restrained twist. In the test set-up of Russel and Burns, the existence of a restrained twist effect was in fact qualitatively indicated. Den Uijl [35] also noticed the effect of a pitch change using push-in and pull-out tests and provided an analytical basis for the presence of a restrained twist effect by comparing bond-slip equations of plain strands and 7-wire strands. Equations expressing the equilibrium of the tendons with their relative twist on one hand and their torsional moment and contact pressure at the steel-concrete interface on the other hand were used for this purpose. It is noted however, that Den Uijl and Russel and Burns only demonstrated the existence of a pitch change effect on the bond behaviour without properly identifying any clear physical explanation whether this is due to the 'lack-of-fit' effect or some other aspect of the mechanical resistance such as concrete interlocking or normal stresses at the steel concrete interface resulting from restrained twist.

A final important aspect of the bond behaviour of a 7-wire strand is the wedging effect which relates to the Poisson's effect of the material. When the strands are tensioned, their cross section reduces due to the Poisson's effect. During de-tensioning, the axial stresses in the strands decrease at the end faces of the element and the axial stress in the strand varies from zero at the face of the element to reach its nominal prestressing value at some distance within the element. This gradual increase of the axial stress also implies that the cross section of the strand will form a wedge towards the end face of the prestressed element (figure 3 ). This wedge action will provide additional interlocking of the strand and will increase the bond strength. The wedge effect is only active along the transfer length $\left(l_{t}\right)$ since the change in the axial strain of the strand only occurs along this length. The influence of the pitch change and of the wedge effect caused by the Poisson's effect are usually combined and referred to in the literature as the Hoyer effect [36].

\subsection{Confinement model}

In the presented approach, the evaluation of the confinement caused by the concrete cover surrounding the strand is done according to a thick-walled cylinder model. In this model, the resistance created by the concrete cover against splitting is obtained by establishing the equilibrium between the radial expansion at the steel-concrete interface and the associated hoop stresses across the cylinder wall. In doing so, the constitutive relationship between radial compressive stress $\sigma_{\text {rr }}$ and radial compressive strain $\epsilon_{\mathrm{rr}}$ is determined for a simple configuration 


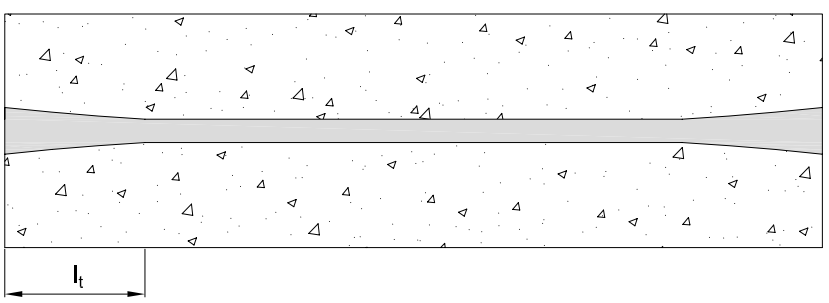

Figure 3: Element side view depicting the strand wedge effect resulting from Poisson's effect

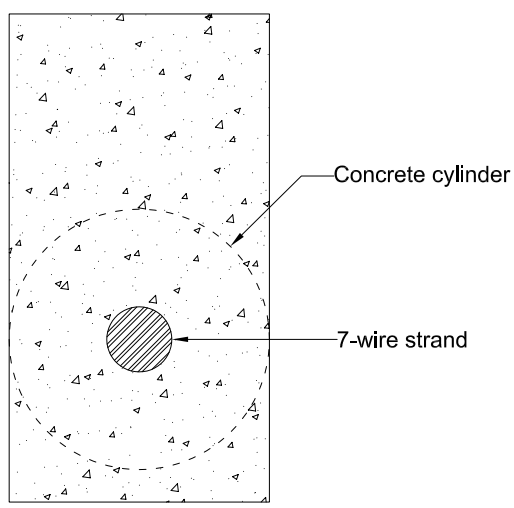

Figure 4: Thick-walled cylinder model

comprising a single prestressing strand (with circular cross section) surrounded by a concrete cylinder (figure 4). The inner radius of this cylinder is determined by the strand radius while the outer radius is defined as the summation of the strand radius and the concrete cover.

Three stages can be defined in the constitutive behaviour (figure 5). The first stage represents the linear elastic behaviour of the concrete cylinder. The radial compressive stress induces circumferential tensile stresses at the interface. This relationship remains linear as long as the hoop stresses surrounding the prestress strand are smaller than the concrete tensile stress. When the hoop stress exceeds the tensile strength, the second stage starts in which radial microcracks initiate at the steel-concrete interface and propagate throughout a now partially cracked concrete cylinder.

The micro-cracking stage can be idealised using a fictitious crack model with a bilinear concrete softening curve. Finally, the cracking front propagates to the outer radius of the concrete cylinder and the constitutive behaviour of a fully cracked concrete cylinder is defined which marks the end of stage 2 . In stage 3 the crackwidth increases, leading to the formation of macro-cracks. This results in further softening of the concrete. By taking into account the non-linear softening behaviour of concrete, the loss of confinement due to the formation of splitting cracks surrounding the prestressing strand can then be taken into account.

\subsection{Bond-slip relation}

As aforementioned, the bond behaviour of 7-wire strands is mainly conditioned by the mechanical resistance of the 7-wire

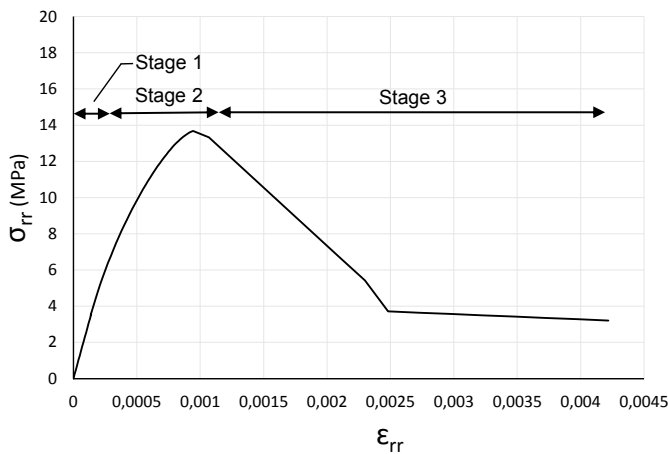

Figure 5: Radial compressive stress - radial compressive strain relation of the thick-walled cylinder model

strand and by the friction at the steel-concrete interface. Tepfers [37] described the bond strength as a function of the radial compressive stress at the steel concrete interface using a dry friction law.

$$
\tau=\tau_{0}+\mu \sigma_{\mathrm{rr}}
$$

With $\tau$ being the bond strength at a particular point along the strand and $\tau_{0}, \mu$ and $\sigma_{\text {rr }}$ defining respectively the initial bond strength, the friction coefficient of the material and the radial stress at the steel-concrete interface. The thick-walled cylinder model describes the constitutive behaviour of the radial compressive stress in function of the radial compressive strain, $\sigma_{\mathrm{rr}}\left(\epsilon_{\mathrm{rr}}\right)$. The evaluation of $\sigma_{\mathrm{rr}}$ in function of $\epsilon_{\mathrm{rr}}$ is the result of the analysis of the thick-walled cylinder model. Keeping in mind the different stages of the model through the incorporation of the non-linear concrete material behaviour, the explicit functions denoting $\sigma_{\mathrm{rr}}\left(\epsilon_{\mathrm{rr}}\right)$ at each seperate stage are quite substantial and are therefore not given in this paper. Reference is made to the work of Den Uijl [24] in which the entire method of establishing this constitutive behaviour is provided.

Considering the fact that the chemical adhesion is negligible, $\tau_{0}$ in equation 1 can be set to zero. The bond strength can now be expressed as;

$$
\tau=\mu \sigma_{\mathrm{rr}}\left(\epsilon_{\mathrm{rr}}\right)
$$

The bond-slip relation can be obtained by evaluating the relation between the radial strain $\epsilon_{\mathrm{rr}}$ and the slip $\delta$. In order to get this relationship, the total radial strain is expressed as the sum of three components.

$$
\epsilon_{\mathrm{rr}, \mathrm{tot}}=\epsilon_{\mathrm{rr}, v}+\epsilon_{\mathrm{rr}, \text { lof }}+\epsilon_{\mathrm{rr}, \mathrm{pitch}}
$$

In this equation, $\epsilon_{\mathrm{rr}, v}, \epsilon_{\mathrm{rr}, \text { lof }}$ and $\epsilon_{\mathrm{rr}, \text { pitch }}$ denote the contributions associated with respectively the wedge effect, the 'lackof-fit' effect and the pitch effect to the total radial strain at the interface. The strain associated with the wedge effect can easily be expressed in function of the slip. The shortening of the prestress strand is represented by the slip $(\delta)$ and the length over which this shortening takes place is the transfer length $\left(l_{\mathrm{t}}\right)$. The axial strain is therefore equal to the slip divided by the transfer 
length. Using Poisson's ratio, the change in axial stress is converted into a radial stress. The increase in radial compressive strain can now be denoted as

$$
\epsilon_{\mathrm{rr}, v}(\delta)=C_{v} v_{\mathrm{s}} \frac{\delta}{l_{\mathrm{t}}}
$$

where $C_{v}$ is a calibration constant, $v_{\mathrm{s}}$ is the steel Poisson's ratio and $l_{\mathrm{t}}$ is the transfer length. The average axial strain of the strand is assumed to be proportional to the ratio between the total slip and the transfer length. This expression forces an iterative procedure to be initiated to characterise the bond-slip behaviour since this behaviour depends on the transfer length which in turn depends on the bond properties. A way of dealing with this aspect of the analysis will be proposed in section 3 .

The contribution of the 'lack-of-fit' effect and pitch effect is less obvious to directly relate with the slip without a deep understanding of their physical background. As a reasonable first approximation, it is opted to combine the influence of the 'lack-of-fit' and pitch effects in one single contribution namely the radial strain associated with the mechanical resistance of the wire $\left(\epsilon_{\mathrm{rr}, \mathrm{mech}}\right)$.

$$
\epsilon_{\mathrm{rr}, \text { mech }}=\epsilon_{\mathrm{rr}, \mathrm{lof}}+\epsilon_{\mathrm{rr}, \mathrm{pitch}}
$$

From the experimental research of Den Uijl [35, 38], it is observed that the normal stresses resulting from the pitch change and 'lack-of-fit' is proportional to the variation of the slip displacement per unit length. Therefore, equation 6 is proposed, defining $\epsilon_{\mathrm{mech}}$ in function of $\delta$.

$$
\epsilon_{\mathrm{rr}, \text { mech }}(\delta)=C_{\text {mech }} \frac{\delta}{l_{\mathrm{t}}}
$$

Where $\delta / l_{\mathrm{t}}$ denotes the mean slip per unit length over the whole transfer length. A second calibration constant characterising the radial strain behaviour resulting from the mechanical properties of the strand $\left(C_{\text {mech }}\right)$ is thus introduced. Setting equations 4,5 and 6 into equation 3 yields the following empirical relation describing the dilation behaviour of the strand in function of the slip.

$$
\epsilon_{\mathrm{rr}, \text { tot }}(\delta)=\left(C_{v} v_{\mathrm{s}}+C_{\mathrm{mech}}\right) \frac{\delta}{l_{\mathrm{t}}}
$$

Combining equation 7 with equation 2 gives an expression of the full bond-slip behaviour at the steel-concrete interface. The values of the friction coefficient $\mu$ and the calibration constants $C_{v}$ and $C_{\text {mech }}$ will be further investigated in section 3 .

It is important to notice here that this bond-slip model should only be applied for strands in a push-in condition. The change in axial strain is indeed considered as having a positive effect on the bond behaviour due to the wedge action that prevents further slip of the strand into the concrete. Therefore, this bondslip model will only yield an appropriate bond-slip behaviour for concrete elements which are prestressed using pre-tensioned strands. Moreover, the model is not suited to describe the bondslip behaviour for general bond tests on small cylindrical specimens (e.g. pull-out bond tests, push-in tests or combined pullout/push-in bond tests, as described by Abrishami [39]). Contrary to the description of small-scale elements by Mitchell et al. [40] and Oh et al. [26], Abrishami used concrete specimens where the length was $150-300 \mathrm{~mm}$. Thus smaller than the transfer length. For these specimens, a proper transfer length cannot be determined simply due to the limited size of the specimen. Therefore, the radial strain-slip relation $\left(\epsilon_{r r}(\delta)\right)$ is no longer valid. The exploitation of the results of these simple bond tests also assume a uniform distribution of the bond stresses at the strand-concrete interface. This is valid due to the limited size of the test specimens. However, this is not the case for a fullscale element. Bond stresses will then only appear when a slip between the concrete and the tendon occurs and the value of the bond strength is dependent on the confining effect of the surrounding concrete. For the remaining of this paper, smallscale specimen refer to specimen for which, even if of limited length, the total length is always larger than the transfer length so that the radial strain-slip relation $\left(\epsilon_{r r}(\delta)\right)$ will be valid.

\section{Bond model: calibration and validation}

In order to calibrate the constants of the proposed bond-slip model, two experimental campaigns performed by Mitchell et al. [40] and Oh et al. [26] are selected. Both campaigns were conducted independently but used a similar test set-up. The transfer lengths of relatively short pre-tensioned beams were analysed using strain gauges along the prestress strands. Several parameters such as the prestressing force, the concrete strength, the concrete cover, and the strand radius were varied. Furthermore, in the experimental study of Oh et al. beams with one or two prestressing strands were examined, allowing the opportunity to investigate the bond-slip model for beams with multiple strands.

\subsection{Summary of the experimental campaign}

The experimental campaigns investigated small pretensioned beams with rectangular cross sections. The ranges of the parameters analysed by Mitchell et al. and Oh et al. can be found in table 1. The experiments of $\mathrm{Oh}$ et al. also included beams with two strands. For these beams the strand spacing was varied between values of $2 d, 3 d$ and $4 d$ with $d$ being the strand diameter. Prestress release was achieved using different methods. Mitchell et al. released the prestress force by slowly reducing the pressure in the hydraulic rams. Oh et al. achieved prestress release by cutting the prestress strands. Both authors do not release the prestress force simultaneously at both ends. The measured transfer length will thus be different at both sides of an element. The side at which the strands are released is called the live end by Mitchell et al. and the cut end by $\mathrm{Oh}$ et al. The following nomenclature is applied for the different experimental beams. The beams of Mitchell et al. are designated using three numbers where the first number denotes the strand radius, the second and third number denote the concrete compressive strength at testing and the embedment length, respectively. The nomenclature of the experiments by $\mathrm{Oh}$ et al. consists of a letter $\mathrm{M}$ or $\mathrm{T}$ depending on whether the beam has one (mono) or two (twin) strands followed by a number denoting the strand radius, 12 for $12.7 \mathrm{~mm}$ and 15 for $15.2 \mathrm{~mm}$ 
Table 1: Value ranges experimental studies bond model

\begin{tabular}{lcc}
\hline Parameter & $\begin{array}{c}\text { Range } \\
\text { Mitchell et al. }\end{array}$ & $\begin{array}{c}\text { Range } \\
\text { Oh et al. }\end{array}$ \\
\hline Prestress force [MPa] & $922-1442$ & $1357-1418$ \\
Concrete strength [MPa] & $31-89$ & $47-61$ \\
Strand radius [mm] & $9.5,12.7,15.2$ & $12.7,15.2$ \\
Concrete cover [mm] & $50,75,100$ & $30,40,50$ \\
\hline
\end{tabular}

strands and another letter $\mathrm{N}$ or $\mathrm{H}$ for normal or high strength concrete. The final two codes denote the concrete cover and the number of the test beam in the series. The methods used for measuring the transfer length also differ for both authors. While both used strain gauges to measure the strain distribution along the strand, Mitchell et al. applies a Slope Intercept (SI) method whereas Oh et al. used an Average Maximum Strain (AMS) method. The SI method is a graphical method in which the transfer length is determined as the distance from the end face of the element to the intersection of two curves fitted against the axial strain measured respectively in the transfer region and beyond this region. A major drawback to this graphical method is that it is to some extent subjective, so different persons can deduce different values for the transfer length with the same experimental data. Furthermore, it relies strongly on the number of data points. The AMS method does not have the drawback of being biased by the person performing the analysis. First, the data values are smoothed out by averaging subsequent data points in order to avoid the influence of locally diverging readings. The transfer length is then conventionally determined as the length required to obtain an axial strand strain of $95 \%$ of the maximum average strain of the data set. The accuracy depends thus on the number of data points and on the precision of the measurements in the zone where the $95 \%$ line intersects the strain curve.

\subsection{Finite element analysis: stage I}

A numerical analyses of the pre-tensioned beam models is performed using the software package TNO DIANA. 8-noded quadrilateral elements are applied to model the concrete and the prestress tendon is modelled using one-dimensional embedded truss elements [41]. Similar to the approach provided by Arab [29], the bond-slip relation presented in section 2.3 is implemented in the material behaviour of the truss by application of a friction stress-slip diagram. Linear elastic material properties are used to model the constitutive behaviour of steel and concrete. The influence of the radial expansion of the strand, possible splitting cracks and softening behaviour on the confining action of the concrete cover and thus on the bond strength, is already implicitly included in the constitutive law of the thickwalled cylinder model and thus must not be included in the material behaviour of the concrete beam.

Only the concrete compressive strength at the time of testing is known from the experimental data. Therefore, estimations are needed for some necessary material parameters. An evaluation of the tensile strength and the Young's modulus is made in compliance with the formulas available in the CEB FIB Model
Code [17]. The following equations are applied to estimate the value of the concrete tensile strength.

$$
\begin{array}{ll}
f_{\mathrm{ctm}}=0.3 \cdot f_{\mathrm{ck}}^{2 / 3} & f_{\mathrm{cc}} \leq 50 \mathrm{MPa} \\
f_{\mathrm{ctm}}=2.12 \ln \left(1+0.1\left(f_{\mathrm{ck}}+\Delta f\right)\right) & f_{\mathrm{cc}}>50 \mathrm{MPa}
\end{array}
$$

With $f_{\text {ctm }}$ the mean concrete tensile strength, $f_{\text {ck }}$ the characteristic concrete compressive strength, $\Delta f$ equals $8 \mathrm{MPa}$ [17] and $f_{\mathrm{cm}}$ is the actual cubic compressive strength at testing. The Young's modulus was evaluated using

$$
E_{\mathrm{ci}}=E_{\mathrm{c} 0}\left(\frac{f_{\mathrm{cm}}}{10}\right)^{1 / 3}
$$

With $E_{\mathrm{ci}}$ the Young's modulus at testing and $E_{\mathrm{c} 0}$, a reference Young's modulus equal to $21.5 \cdot 10^{3} \mathrm{MPa}$.

The prestress force is modelled as an external force acting on the end nodes of the prestress tendon at the level of the end faces of the beam. This force will then progressively be transferred from the prestress tendon to the surrounding concrete via the bond stresses at the steel-concrete interface. However, this modelling methodology requires the superposition of the initial stress and strain in the tendon (i.e. stress and strain corresponding to the pre-tensioning phase of the tendon before pouring of the concrete) to the values resulting from the finite element analysis, in order to achieve the actual total axial stress- and strain-field in the tendon. The non-linear analysis is performed using a full Newton-Rhapson solution algorithm. An example of a pre-tensioned beam model with the applied boundary conditions can be seen in figure 6 . The black line in the beam depicts the prestress strand. The corner nodes are restricted in the $\mathrm{X}$ - and Z-direction and the bottom nodes in the middle of the beam are blocked in the Y-direction in order to prevent any rigid body motion.

As mentioned in section 2.3, an iterative procedure was followed to obtain the correct bond-slip relation. Figure 7 represents the flowchart of this procedure. In order to compute the bond-slip behaviour, the transfer length has to be known (equation 4). However, this transfer length is dependent on the bondslip behaviour and therefore, it cannot be evaluated accurately in the first iteration step. An initial guess is made to evaluate the bond-slip relation. Next, this relation is implemented in the interface behaviour of the finite element model and the analysis is performed. From the analysis results, the strain field of the beam is plotted and a new transfer length $\left(l_{t, i+1}\right)$ is computed following the $95 \%$ AMS method. If this updated value does not match the previous value of the transfer length $\left(l_{t, i}\right)$, the new value of the transfer length is used to re-evaluate the bond-slip behaviour and the analysis is performed again until convergence of the transfer length is reached. The initial guess is chosen equal to 60 times the diameter of the 7-wire strand in accordance with AASHTO regulations [14].

In order to take into account the presence of multiple strands in a single cross section, the concrete cover applied in the thickwalled cylinder model is replaced by an effective concrete cover $\left(c_{\text {eff }}\right)$. This effective concrete cover is an average of the spacing between the strands and the concrete cover. In this regard, a single value for the concrete cover which effectively represents 


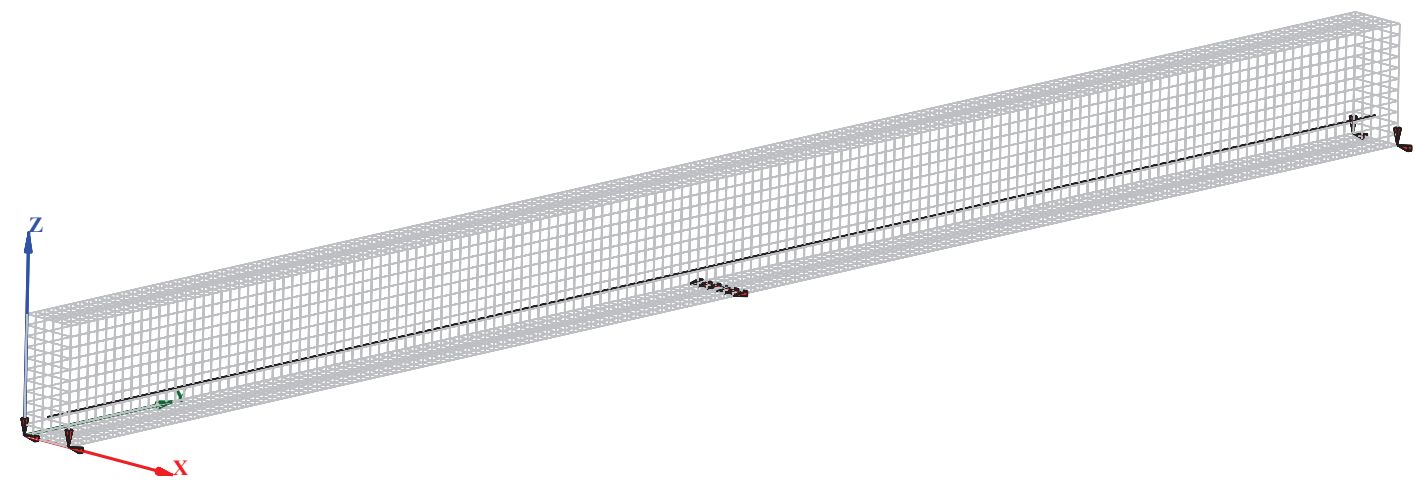

Figure 6: M15-N-C4 beam model with details of the applied boundary conditions

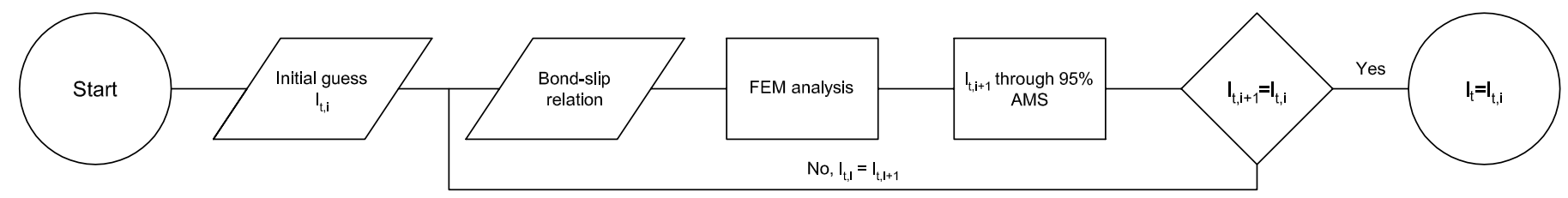

Figure 7: Flowchart of the bond-slip model

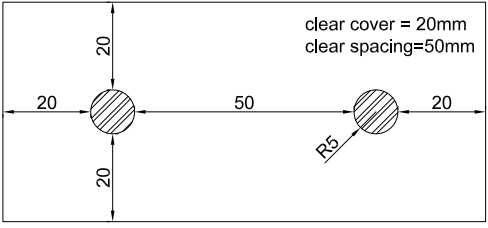

Original values for concrete cover and clear strand spacing

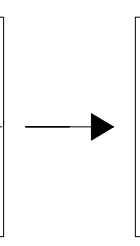

Re-evaluated effective cover
Figure 8: Example of the evaluation of the effective concrete cover for a cross section which is prestressed with multiple strands

all strand throughout the element is obtained. When a beam with multiple strands is analysed, $c_{\text {eff }}$ is computed first. The evaluation of the bond-slip behaviour is then performed using a model with only one strand with a concrete cover equal to $c_{\text {eff }}$. The following formula is used to calculate an effective concrete cover $\left(c_{\text {eff }}\right)$.

$$
c_{\mathrm{eff}}=\frac{2 c_{\mathrm{s}}+(n-1) s}{2 n}
$$

Where $c_{\mathrm{s}}$ represents the concrete cover to the faces of the beam, $n$ is the amount of strands and $s$ is the strand spacing. An example is given in figure 8 . The specimen is prestressed with two strands. The clear concrete cover is $20 \mathrm{~mm}$ and the strand spacing is $50 \mathrm{~mm}$. Evaluating equation 10 in the horizontal and vertical direction returns an effective horizontal cover $c_{\text {eff1 }}$ of $22.5 \mathrm{~mm}$ and an effective vertical cover $c_{\text {eff } 2}$ of $20 \mathrm{~mm}$.

\subsection{FEA results: model calibration}

A total of 22 pre-tensioned beams were modelled according to the analysis procedure described in the previous section. Ten
Table 2: Calibrated values for the constants of the bond-slip model

\begin{tabular}{lr}
\hline Calibration constant & Value \\
\hline$\mu$ & 0.75 \\
$C_{v}$ & 1 \\
$C_{\text {mech }}$ & 0.20 \\
\hline
\end{tabular}

beams are selected from the experimental study of Mitchell et al. and twelve from $O$ h et al's campaign. The ten beams from Mitchell et al. are used to first calibrate $\mu, C_{v}$ and $C_{\text {mech. }}$ The remaining twelve beams of $\mathrm{Oh}$ et al. are then used to validate the model by comparing the experimental results with the results of a direct numerical analysis making use of these calibrated values of the constants.

For the calibration of the bond-slip model, it is assumed that $\mu, C_{v}$ and $C_{\text {mech }}$ are constant. The values of the calibration constants are determined by performing the bond-slip analysis using different values for each coefficient while the other ones remain unchanged. The resulting value of the transfer length is then evaluated by comparing it to the experimental value of the corresponding specimen and the appropriate values for $\mu, C_{v}$ and $C_{\text {mech }}$ are identified.

Figure 10 illustrates the impact on the transfer length for different values of the individual calibration constants. For each graph, the value of a single calibration constant is varied while the others calibration values are kept constant. It can be seen that the impact of $C_{v}$ and $C_{\text {mech }}$ on the transfer length values is limited in comparison with that of $\mu$.

The values for the calibration constants can be found in table 2 . These values provide good agreement for all ten beams 


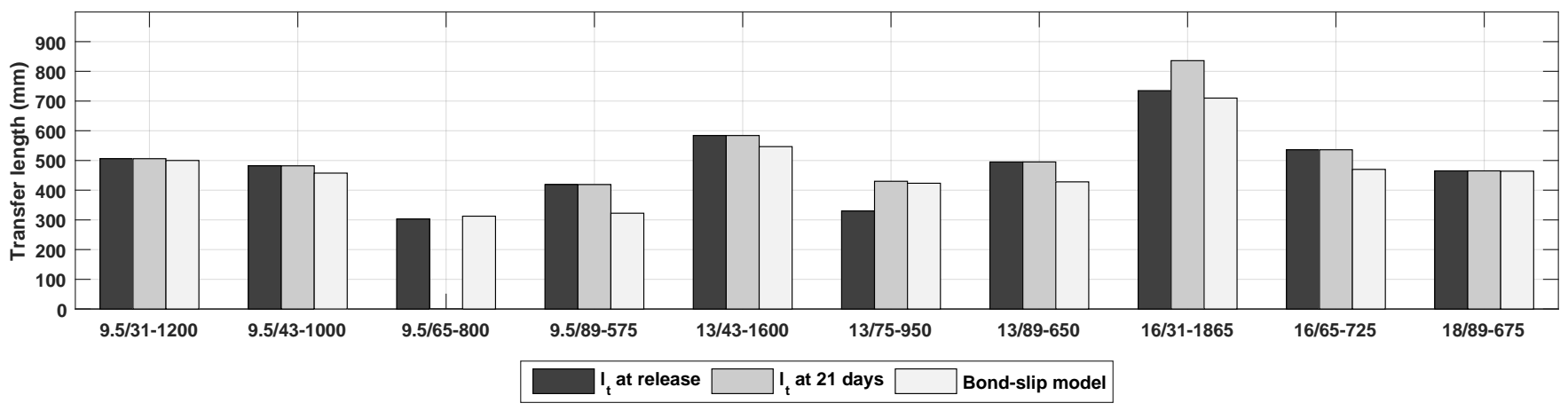

(a) Mitchell et al.

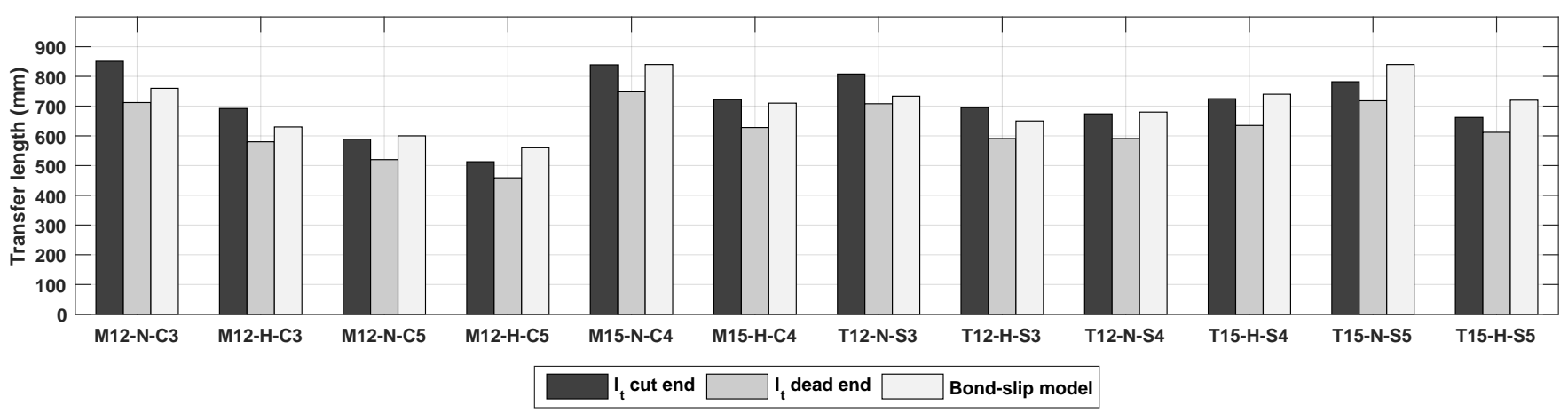

(b) Oh et al.

Figure 9: Comparison experimental results with bond-slip model

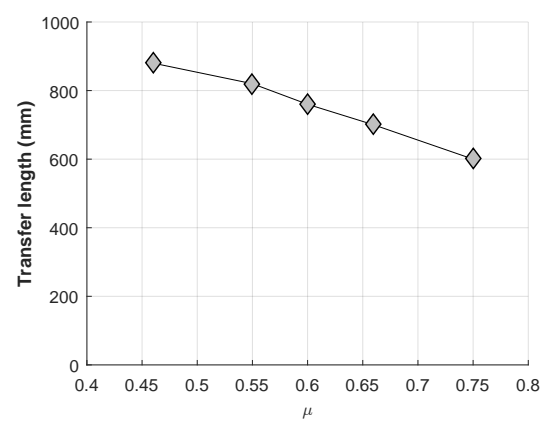

(a) Influence of $\mu$

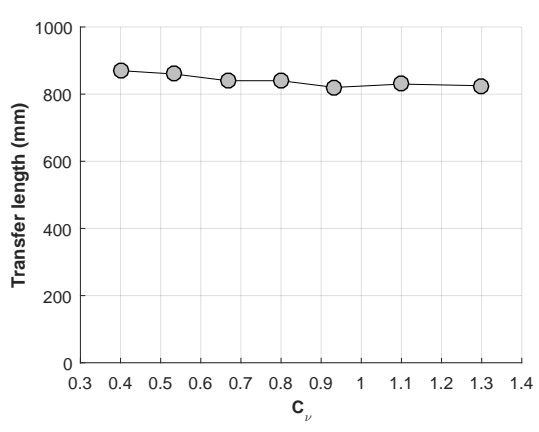

(b) Influence of $C_{v}$

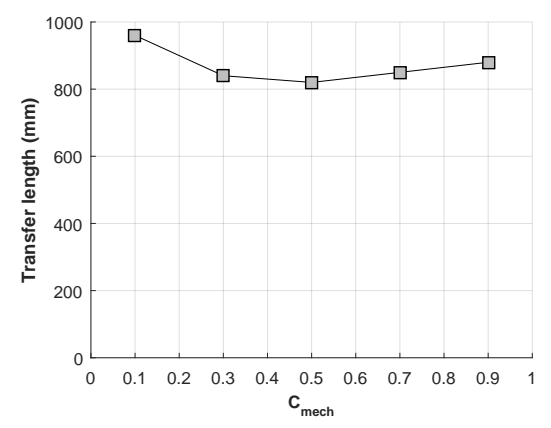

(c) Influence of $C_{\text {mech }}$

Figure 10: Impact of calibration constants on computed transfer length values 
taken from the experimental campaign of Mitchell et al. assuming a constant value for $\mu, C_{v}$ and $C_{\text {mech }}$ across all the models. A value of 0.75 for $\mu$ has been suggested for concrete by other authors [29, 30]. Throughout the literature, friction coefficients ranging between 0.4 to 0.7 [32, 37, 29] can be found. Some authors even use values up to and higher than 1 but these models often have an adaptive variable $\mu$ applying for instance a plasticity model for $\mu$ or a slip-dependent friction coefficient $[32,21]$.

Keeping in mind equation 7, the cross-effect of $C_{v}$ and $C_{\text {mech }}$ can clearly be identified. As long as the expression between the brackets remains constant, the same dilation behaviour of the strand can be achieved for different values of $C_{v}$ and $C_{\text {mech. In }}$ order to identify the proper value of the calibration constants, an additional experimental campaign by Bodapati et al. [42] was selected. The prestress tendons in this experimental program were smooth bars hence eliminating the effect of the radial dilation behaviour due to mechanical action of strands. From these experiments, it is concluded that the Poisson's coefficient of steel (0.3) best describes the wedge effect of the strand at the interface which fixes the value of $C_{v}$ at 1.

Finally, based on the assumptions made in section 2.3, stating that the radial expansion caused by the mechanical resistance is proportional to the variation of the slip per unit length and looking to equation 6 , it can be said that the confining action of the mechanical resistance (the 'lack-of-fit' effect and pitch effect) contributing to the frictional behaviour at the steel concrete interface is comparable to that of a radial dilation equal to $20 \%$ of the slip. Therefore, the selected value of the calibration constant $C_{\text {mech }}$ concerning the mechanical resistance is set equal to 0.2 .

The calibrated relation between the radial strain of the sevenwire strand and the slip is represented by

$$
\epsilon_{\mathrm{rr}, \mathrm{tot}}(\delta)=\left(v_{\mathrm{s}}+C_{\text {mech }}\right) \frac{\delta}{l_{\mathrm{t}}}
$$

with $v_{\mathrm{s}}$ equal to 0.3 and $C_{\text {mech }}$ equal to 0.2 .

\subsection{FEA results: model validation}

The transfer length of the bond-slip models was evaluated according to the AMS method. Figures $9 \mathrm{a}$ and $9 \mathrm{~b}$ show a comparison of the experimental results with the results computed using the bond-slip model and the values presented in table 2 . The nomenclature used to denote the various beams in the graphs is explained in section 3.1.

A decent agreement is reached between the results of the bond-slip model and the experimental results of Mitchell et al. The difference can partially be explained by the fact that a different method is used to determine the transfer length. The slope intersect method applied for these experimental results is susceptible to errors due to the subjective interpretation of the data, as mentioned in section 2 . The numerical results should also be compared with the transfer length at release as the material characteristics entered in the model actually correspond to this stage. It appears however that, in some cases, the numerical model comes closer to the value of the transfer length

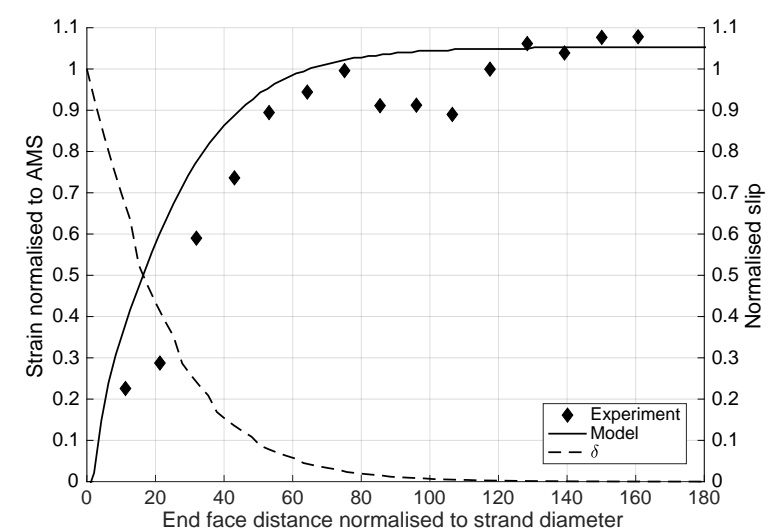

(a) $9.5 / 31 / 1200$ beam

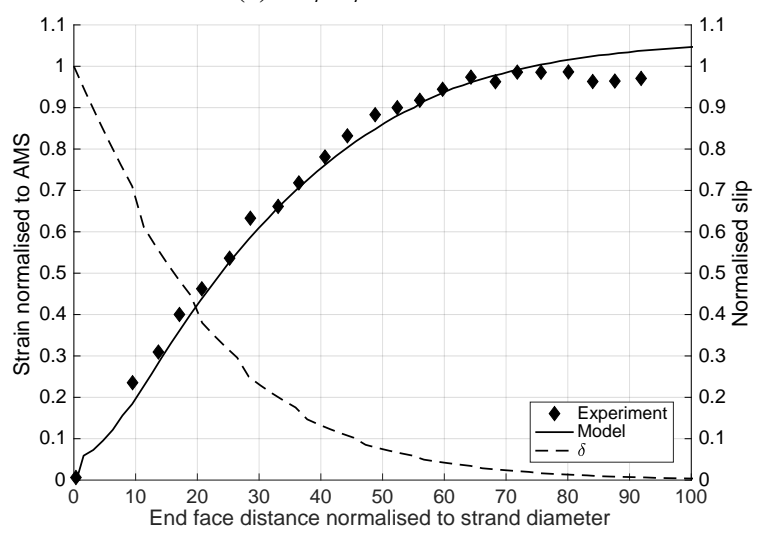

(b) M12-N-C3-2 beam

Figure 11: Comparison prestress build-up in prestress strands

at 21 days (for instance for beam 13/75-950). This could be due to a less accurate measurement at release or to the fact that the intended concrete tensile strength of the beam was not yet reached at release.

The differences can also be explained from the assumptions at the numerical side, the material characteristics in the model are evaluated using formulas 8 and 9 in order to take into account the natural evolution of the Young's modulus and the concrete tensile strength through time. These assumptions might be conservative or not in compliance with actual the material characteristics at the time of testing. Furthermore, the assumption made in section 2.3 (i.e. the mechanical resistance of the strand defined using a linear relationship between the radial displacement and the slip, equation 6) may be overly simplistic. The values of the transfer length obtained by the bond-slip model show good agreement with the experimental results of Oh et al. probably due to the fact that the AMS method was used both in the experimental campaign and in the processing of the numerical data. It is expected that the bond-slip model provides a value for the transfer length between the cut end and dead end values of the experimental campaign. When the strand is cut, certain dynamic effects will occur at the cut end which cause a higher value of the transfer length. Also, after cutting of the prestress tendon, the specimen will move towards the strand anchor point at the dead end side thus also causing prestress release on that 
Table 3: Girder properties

\begin{tabular}{lccc}
\hline & Tx28 & Tx46 & Tx70 \\
\hline Height $(\mathrm{mm})$ & 711 & 1169 & 1778 \\
\# prestressing strands & 36 & 48 & 50 \\
Total prestress force $(\mathrm{kN})$ & 7526 & 8700 & 8700 \\
\hline
\end{tabular}

side. However, some frictional forces can occur between the beam and the casting bed. As a result, the full prestress load might not be released at the dead end. Taking into account the results from the analyses for all 22 beams, the average relative difference between the computed and the experimental values of the transfer length is about $8 \%$. The numerical results were evaluated in reference to experimental transfer length values at release for Mitchell et al. and in reference to the average between the cut end and dead end values for the experimental results of Oh et al.

\subsection{Transfer of prestress force}

Besides the transfer length itself, a further insight can also be given on the strain and slip distribution along the transfer length. Figure 11 shows the numerical results of the axial strain normalised to the AMS strain at the transfer length and the normalised slip in the prestress strand. The increase of the axial stress is compared with to the normalised experimental values of the 9.5/31/1200 beam taken from the experimental campaign of Mitchell et al. and the M12-N-C3-2 beam from Oh et al. Slip values are not available for in the experimental results of either Mitchell et al. or Oh et al.

Good agreement is reached with the experimental results. The difference between the experiment and the numerical model for the 95/31/1200 beam can be justified by the fact that the strains are normalised to the transfer length evaluated by the AMS method for the numerical value while the SI method was used for the experimental value. A better approximation might be reached by including more experimental data points but these were unavailable. Furthermore, a global overestimation of the strain build-up is visible while a good approximation is reached for data points located beyond the transfer length.

The M12-N-C3-2 beam shows very good fitting of the numerical and of the experimental values regarding the increasing part of the curve. The stabilised value seems however to slightly diverge from the numerical value.

\section{Finite element analysis: stage II}

In this second stage of the analysis, the previously calibrated bond-slip model is used to predict the non-linear stress distribution and possible cracking of the anchorage zone of full-scale prestressed girders. Girders with I-shaped cross sections are analysed, namely Tx28-, Tx46- and Tx70-girder (figure 12). Table 3 gives the dimensions, number of prestress strands and total prestressing force for each of the Tx-girders. The numerical results will be validated by comparison with strain data collected from the work of O'Callaghan [5].
The analysis will be conducted in two stages. The first stage evaluates the correct bond-slip model and transfer length of the strands using the analysis procedures discussed in section 3 (figure 7). This will provide a model for the transfer of the prestress force, taking into account the actual geometric and material properties. This bond-slip behaviour is then implemented in a full-scale model in which the non-linear behaviour of concrete is taken into consideration in order to allow for the possible occurrence of crack formation, concrete softening and redistribution of the stresses after cracking.

\subsection{Finite element model}

A representation of the Tx70-girder model can be found in figure 13. 8-noded quadrilateral elements and one-dimensional truss elements are used to respectively model the concrete and steel elements. In contrast with other models available in the literature, which either apply crude simplifications of the transfer of the prestress force or resort to numerically demanding analyses in which the bond-slip behaviour is coupled with the concrete non-linear material behaviour. The analysis approach presented here considers a bond-slip model which is fully decoupled from the non-linear material model in the concrete elements of the anchorage zone. The non-linear concrete material behaviour is taken into account to model the global effect of bursting and spalling stresses on the end-zone reinforcement. Since the prestress strands are modelled with embedded truss elements, no normal behaviour (radial expansion) of the strands and possible splitting cracks are explicitly represented in the model. However, the loss of confinement due to these cracks is correctly included in the bond-slip law via the properly calibrated thick-walled cylinder approach of the first modelling stage. It must be safeguarded however, that at the local level of the concrete material surrounding the prestress strands, the effect of concrete softening is not taken into account twice (once via the concrete material behaviour and once through the bond model). This is ensured through the use of the embedded modelling technique applied to represent the strands. For embedded modelling, the stiffness in surrounding elements is increased to take into account the influence of the reinforcement. The frictional behaviour resulting from the bond-slip analysis is achieved through nodal constraints and master-slave connections between the nodes of the concrete elements and truss elements. Combined with the compressive prestress load, this will result in a compressive state of the continuum elements surrounding the truss elements and interaction between effects of the non-linear material behaviour and the bond-slip model is avoided. The uncoupled method between the bond-slip model in stage I and the full scale model in stage II significantly minimises the numerical demand while maintaining a realistic approximation of the transfer of the prestress force. Due to the bond-slip analysis in stage I, it is not necessary for the concrete non-linear material to be implemented to correctly model the transfer of the prestress force in stage II. Here however, the concrete softening has been taken into account but solely to model the global cracking behaviour in the anchorage zone associated with bursting and spalling. The risk of taking the softening behaviour into account twice is eliminated by ensuring 


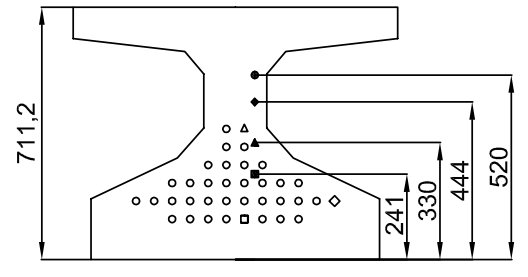

- R3C7 $\diamond \mathrm{R} 4 \mathrm{C} 2 \triangle \mathrm{R} 8 \mathrm{C} 7$

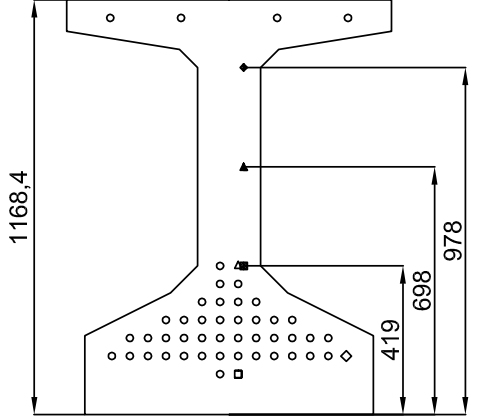

- $\mathrm{R} 2 \mathrm{C} 7 \diamond \mathrm{R} 3 \mathrm{C} 1 \Delta \mathrm{R} 8 \mathrm{C} 7$

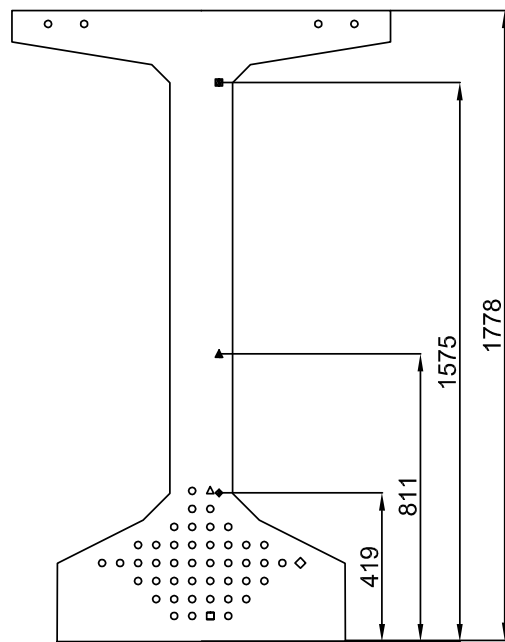

- $\mathrm{R} 1 \mathrm{C} 7 \diamond \mathrm{R} 4 \mathrm{C} 2 \triangle \mathrm{R} 8 \mathrm{C} 7$

(b) $\mathrm{Tx} 46$

(a) $\mathrm{T} \times 28$

(c) $\mathrm{Tx} 70$

Figure 12: Dimensions of experimental girders, prestress tendon layout and location of strain gauges (units: mm)

that the continuum elements surrounding the prestress strands are in compression through the embedded modelling technique.

A rotating smeared crack model with a linear softening curve is applied to analyse the tensile behaviour of the concrete elements in the anchorage zone. The compression curve is in accordance with the CEB FIB 2010 Model Code [17]. The passive reinforcement of the anchorage zone is modelled using embedded truss elements with perfect bond behaviour and linear elastic material behaviour. To minimise the computational load, the model is limited to a quarter of the girder with double symmetric boundary conditions. Non-linear concrete material behaviour is only considered along a length of 1.5 times the transfer length as evaluated by the bond-slip model (represented by the dark gray area in figure 13a). The bond-slip behaviour of the strands is implemented similarly as in the small beam models. Equation 10 is used to calculate two values for the effective concrete cover. One value is used for all strands located at the bottom flange while a second one is applied for the prestress reinforcement situated in the top flange of the girder, since the values of the concrete cover and prestress force are significantly different for these two groups of strands. It would theoretically be possible to evaluate a specific bond behaviour for each strand separately but, considering the small differences in concrete cover between the strands respectively in the lower and upper flange, it is assumed that a reasonable approximation of the global bond behaviour is achieved with an averaged behaviour per zone.

The full beam models include two loading phases. The first one consists in the application of the selfweight while in the second phase, all the prestress loads are applied according to the sequence in which the strands are de-tensioned or flamecut in the production plant. In order to properly account for the self-weight and to model the upwards curvature occurring during the prestressing of the girder, the casting bed is explic-

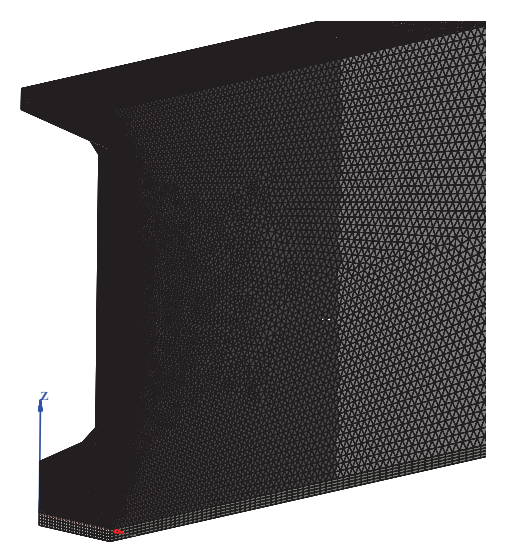

(a) Tx70-girder: mesh

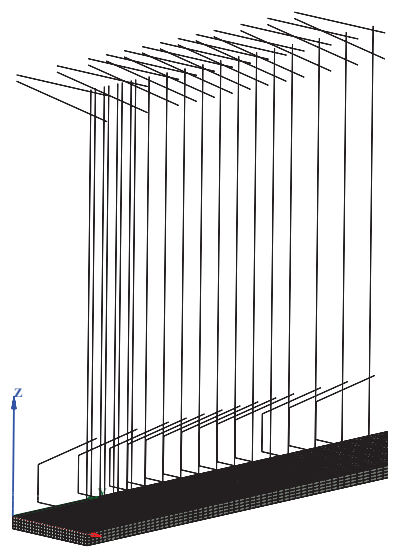

(b) Tx70-girder: reinforcement lay-out

Figure 13: Finite element model: Tx70-girder 
itly modelled by rigid elements and non-linear interface elements are implemented between the girder and the casting bed. The shear stiffness of the interface elements is kept low to fit the reduced friction between the bed and the freshly manufactured girder. The normal compression stiffness at the interface is high in comparison with the tension stiffness to simulate a quasi-unilateral contact. The actual behaviour of the set-up in the manufacturing plant can thus be represented in an accurate way. The model allows for the girder to bend upwards while the downwards bending is prevented by the interface reacting in compression. A sensitivity study was performed to define the values of the normal stiffness in both directions and to assess its influence on the beam response. However, the impact of the changes in normal stiffness on the strain behaviour within the anchorage zone appears to be limited.

\subsection{Full scale girder analysis results}

The results of the finite element model are compared with strain data obtained from the work of O'Callaghan [5]. Figure 14 shows a comparison of the axial strain in several tendons. The locations of the strands are shown in figure 12. The ultimate strain at full transfer as well as the value of the transfer length (i.e. the total length from which a stabilised strain state is reached) is correctly approximated. However, the axial strain seems to increase at a higher rate in the numerical model than is experimentally measured by O'Callaghan. As stated in section 3.2, equation 10 is evaluated to average the bond properties of a set of strands into a single set of bond parameters applied to all strands of the group. In reality, each strand is characterised by its own transfer length, which may explain the differences in strain behaviour when focussing on one given strand. Moreover, the assumption of a linear slip-strain relation, is also likely to have consequences on the simulation of the load transfer mechanism at the local level of a strand.

Furthermore, strain values in the passive reinforcement of the anchorage zone (figure 12) were collected from the experimental data and compared to the numerical values as presented in figure 15.

The numerically predicted strain distribution compares in general well with the experimental measurements. Some discrepancy is however observed for the strain gauges located at $440 \mathrm{~mm}$ in the Tx28-girder (strain associated with the bursting effect). The strains near the end faces of the girders also appear to be slightly underestimated at a height of $698 \mathrm{~mm}$ and $811 \mathrm{~mm}$ for the Tx46- and Tx70-girder respectively (strain associated with the spalling effect).

These discrepancies in the strain field can occur due to the material model. The applied softening model is a total strain crack model, meaning that the strains associated with fracture of the concrete are smeared out over each affected finite element. This influence is assumed to be limited thanks to the application of a fine mesh in the anchorage zone. However, in the bursting and spalling zones, highly sensitive to cracking, this remains limited in the perspective of perfectly capturing a strain concentration associated to the opening of a discrete crack. This could be improved by for instance implementing a discontinu- ous crack model using interface elements at the expected crack locations, provided these could be somehow predicted.

The experimental values also provide discrete strain readings at certain positions. It is very difficult to assess the exact value in the anchorage due to the intricate stress behaviour. The small difference in the experimentally and numerically determined strain values in the prestress tendons visible in figure 14 can also indicate a slightly different load transfer. This could lead to a change in load transfer, crack location and post-crack stress redistribution in the anchorage zone causing the discrepancies visible in figure 15. Moreover, differences between the values provided by the numerical modelling environment and the real life practical specimens are always expected to some extent.

Based on the proposed FEM methodology, an optimisation of the passive reinforcement considering efficient and economical end-zone detailing under the prestress load can be performed.

\section{Conclusion}

In this paper, an innovative two-stage modelling strategy is proposed, aiming at the prediction of the non-linear strain and stress distribution in the anchorage zone of pre-tensioned, concrete girders. The approach consists of a decoupled analysis. In the first stage, the transfer length and the bond-slip behaviour of one prestress tendon is characterised by evaluating the confinement effect associated with the concrete surrounding the strand and by deriving a specific relation describing the radial strain $\epsilon_{r r}$ in function of the slippage of the strand $\delta$. In the second stage, this bond-slip relation is applied in a full-scale finite element analysis of the girder. In contrast with other available full-scale models, no experimental input is required and no limiting assumptions are made regarding the transfer mechanism of the prestress force. The modelling approach is able to accurately evaluate the progressive global transfer of the prestress force and to assess the subsequent non-linear strain distribution in the anchorage zone. Both the numerical evaluation of the bondslip behaviour and of the strain fields of the anchorage zone models were validated with experimental results, showing an acceptable level of accuracy against a reasonable numerical investment. The whole procedure can be carried out entirely on the basis of geometric and material properties available at the design stage. Therefore, it can be efficiently used to analyse the strain field in the anchorage zone and further to verify and optimise the scheme and amount of passive reinforcement in the anchorage zone.

\section{Acknowledgement}

The support of this research by the Bijzonder Onderzoeksfonds Doctoral Funding program of Hasselt University is gratefully acknowledged.

\section{References}

[1] C. Tuan, S. Yehia, N. Jongpitaksseel, M. Tadros, End zone reinforcement for pretensioned concrete girders, PCI Journal (2004) 16. 
[2] P. Okumus, M. Oliva, Finite element analysis of deep wide-flanged pre-stressed girders, Tech. rep., Wisconsin Highway Research Program (2011).

[3] P. Okumus, M. G. Oliva, S. Becker, Nonlinear finite element modeling of cracking at ends of pretensioned bridge girders, Engineering Structures 40 (2012) 267-275. doi:10.1016/j.engstruct.2012.02.033.

[4] P. Okumus, M. G. Oliva, Evaluation of crack control methods for end zone cracking in prestressed concrete bridge girders, PCI Journal 58 (2013) 91-105.

[5] M. R. O'Callaghan, Tensile stresses in the end regions of pretensioned i-beams at release, Thesis, University of Texas, Texas (2007).

[6] American Concrete Institute, ACI concrete terminology (2013).

[7] J. C. Galvez, J. M. Benitez, B. Tork, M. Casati, D. Cendón, Splitting failure of precast prestressed concrete during the release of the prestressing force, Engineering Failure Analysis (16) (2009) 2618-2634.

[8] J. Marti-Vargas, P. Serna, J. Navarro-Gregori, L. Pallarés, Bond of 13 mm prestressing steel strands in pretensioned concrete members, Engineering structures 41 (2012) 403-412.

[9] J. M. Benitez, J. C. Galvez, M. Casati, Study of bond stressslip relationship and radial dilation in prestressed concrete, International Journal for Numerical and Analytical Methods in Geomechanicsdoi:10.1002/nag.2158.

[10] A. Torre-Casanova, L. Jason, L. Davenne, X. Pinelli, Confinement effects on the steel-concrete bond strength and pull-out failure, Engineering Fracture Mechanics 97 (2013) 92-104. doi:10.1016/j.engfracmech.2012.10.013.

[11] R. Gustavson, Experimental studies of the bond response of three-wire strands and some influencing parameters, Materials and Structures (37) (2004) 96-106.

[12] D. Moon, Z. Goanseup, K. Jang-Ho, L. Seung-Jung, K. Gyuseon, On strain change of prestressing strand during detensioning procedures, Engineering Structures 32 (2010) 2570-2578.

[13] J. Marti-Vargas, P. Serna, J. Navarro-Gregori, J. Bonet, Effects of concrete compusition on transmission length of prestressing strands, Construction and Building Materials 27 (2012) 350-356.

[14] AASHTO, Standard specifications for highway bridges, Tech. rep., American Association of State Highway and Transportation Officials inc (1996).

[15] Committee 318, American Concrete Institute, Building code requirements for structural concrete (2014)

[16] European Committee for Standardization, Eurocode 2: Design of concrete structures - part 1-1: General rules and rules for buildings (+ ac:2008) (2005).

[17] Fédération internationale du béton, Comité euro-international du béton, Fédération internationale de la précontrainte, Model code 2010: first complete draft., Fédération internationale du béton, Lausanne, Switzerland, 2010

[18] R. W. Floyd, M. B. Howland, W. M. Hale, Evaluation of strand bond equations for prestressed members cast with self-consolidating concrete, Engineering Structures 33 (10) (2011) 2879-2887. doi:10.1016/j.engstruct.2011.06.012.

[19] A. Ayoub, F. C. Filippou, Finite-element model for pretensioned prestressed concrete girders, Journal of structural engineering 136 (4) (2009) 401-409.

[20] H. Tabatabai, T. Dickson, the history of the prestressing strand development length equation, PCI Journal 38 (1993) 64-75.

[21] K. Lundgren, J. Magnusson, Three-dimensional modeling of anchorage zones in reinforced concrete, Journal of engineering mechanics 127 (7) (2001) 693-699.

[22] J. C. Galvez, J. M. Benitez, M. J. Casati, B. S. Tork, D. A. Cendon, Cohesive-frictional model for bond and splitting action of prestressing wire, International Journal for Numerical and Analytical Methods in Geomechanics 35 (11) (2011) 1257-1277. doi:10.1002/nag.956.

[23] S. Timoshenko, Strength of Materials part 2, Lancaster Press, 1941.

[24] J. A. Den Uijl, A. J. Bigaj, A bond model based on ribbed bars on concrete confinement, Tech. rep., Delft University of technology, Delf (1996).

[25] J. Fellinger, Shear and Anchorage Behaviour of Fire Exposed Hollow Core Slabs, DUP Science.

[26] B. Oh, E. Kim, Y. Choi, Theoretical analysis of transfer lenghts in pretensioned prestressed concrete members, Journal of Engineering Mechanics 132 (2006) 1057-1066.
[27] J. M. Benitez, J. C. Galvez, Bond modelling of prestressed concrete during the prestressing force release, Materials and Structures 44 (1) (2010) 263-278. doi:10.1617/s11527-010-9625-5.

[28] A. Abdelatif, J. Owen, M. Hussein, Modelling the prestress transfer in pre-tensioned concrete elements, Finite Elements in Analysis and Design 94 (2015) 47-63.

[29] A. A. Arab, S. S. Badie, M. T. Manzari, A methodological approach for finite element modeling of pretensioned concrete members at the release of pretensioning, Engineering Structures 33 (6) (2011) 1918-1929. doi:10.1016/j.engstruct.2011.02.028.

[30] A. A. Arab, Finite element modeling of pretensioned concrete girders: A methodological approach with applications in large strands and end zone cracking (2012).

[31] J. Marti-Vargas, P. Serna, W. Hale, Strand bond performance in prestressed concrete accounting for bond slip, Engineering Structures 51 (2013) 236-244.

[32] Fédération international du Béton, Bullettin 10 bond of reinforcement, Tech. rep., International federation for structural concrete, (2000).

[33] M. Stocker, M. Sozen, Investigation of prestressed reinforced concrete hof highway bridges. part vi: Bond characteristics of prestressing strand., Tech. rep., University of Illinois (1969).

[34] B. Russell, N. Burns, Design guidelines for transfer, development and debonding of large diameter seven wire strands in pretensioned concrete girders, Tech. rep., State Department of Highways and Public Transportation (1993).

[35] J. A. Den Uijl, Tensile stresses in the transmission zones of hollow-core slabs, Tech. Rep. 5-83-10, Delft University of technology, Stevin Laboratories (1983).

[36] V. Briere, K. Harries, J. Kasan, C. Hager, Dilation behavior of seven-wire prestressing strand the hoyer effect, Construction and Building Materials 40 (2013) 650-658.

[37] K. Lundgren, Three-dimensional modelling of bond in reinforced concrete: theoretical model, experiments and applications, Chalmers tekniska hgsk., Göteborg, 1999.

[38] J. Den Uijl, Bond modelling of prestressing strand, ACI Special Publication; A tribute to Peter Gergely 180 (1998) 145-169.

[39] H. H. Abrishami, D. Mitchell, Analysis of bond stress distributions in pullout specimens, Journal of Structural Engineering 122 (3) (1996) 255-261.

[40] D. Mitchell, W. Cook, A. Khan, T. Tham, Influence of high strength concrete on transfer and development length of pretensioning strand, PCI Journal 38 (1993) 52-66.

[41] DIANA (release 9.5) User's Manual Element Library, Delftechpark 19a, Delft, The Netherlands, 2014.

[42] N. Bodapati, W. Zhao, R. Peterman, C. Wu, B. Beck, M. Haynes, J. Holste, Influence of indented wire geometry and concrete parameters on the transfer length in prestressed concrete crossties, Proceedings of the 2013 Joint Raill Conference April 15-18. 


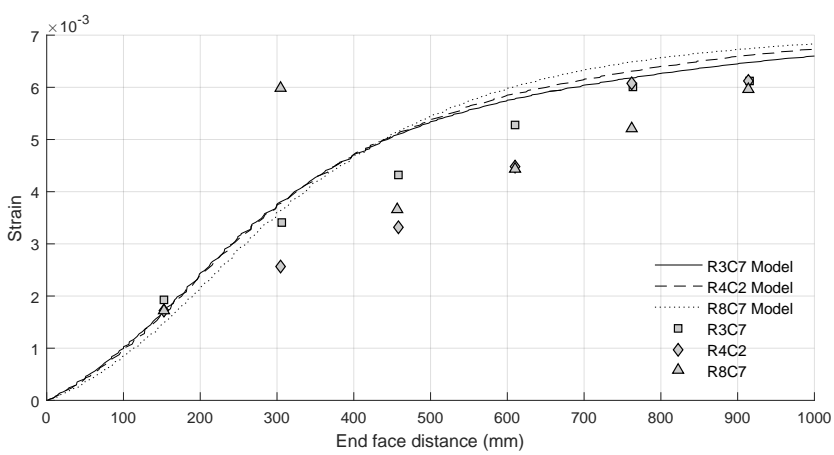

(a) Tx28-girder

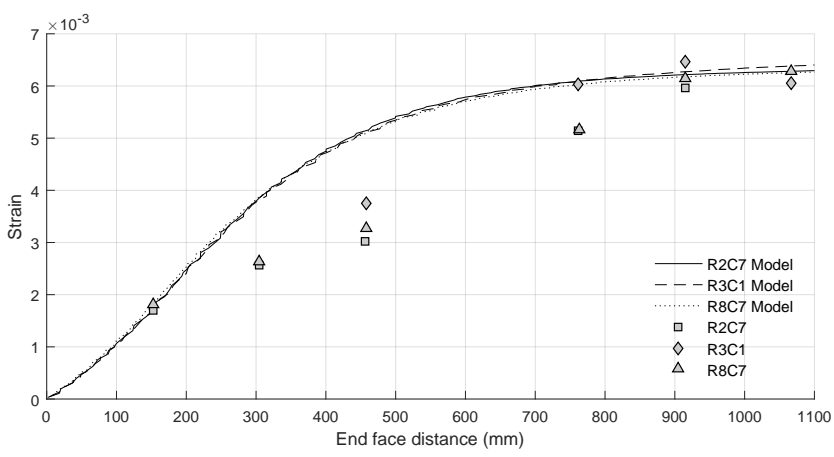

(b) Tx46-girder

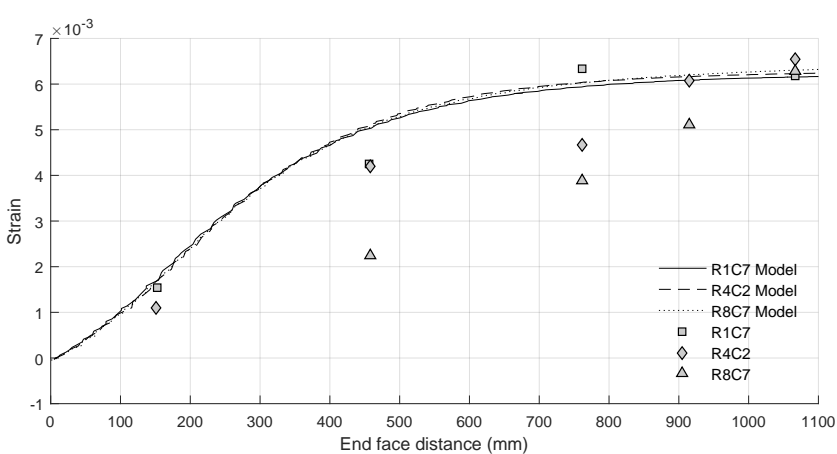

(c) Tx70-girder

Figure 14: Comparison numerical axial tendon strains with experimental results of Tx-girders

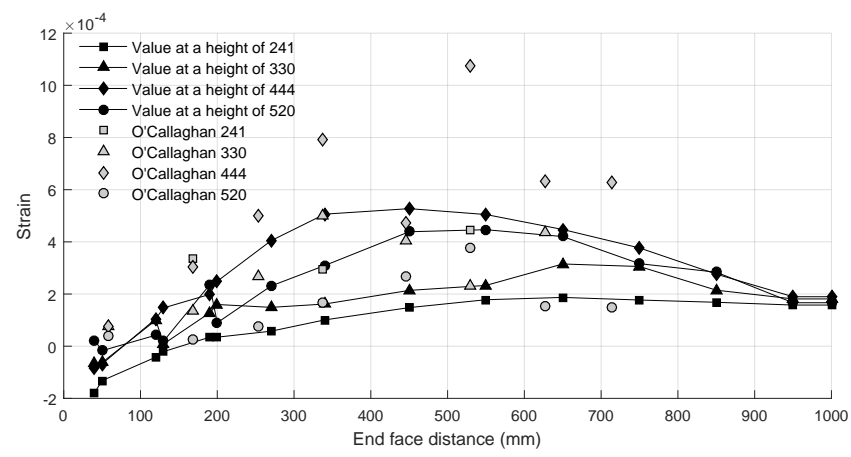

(a) Tx28-girder

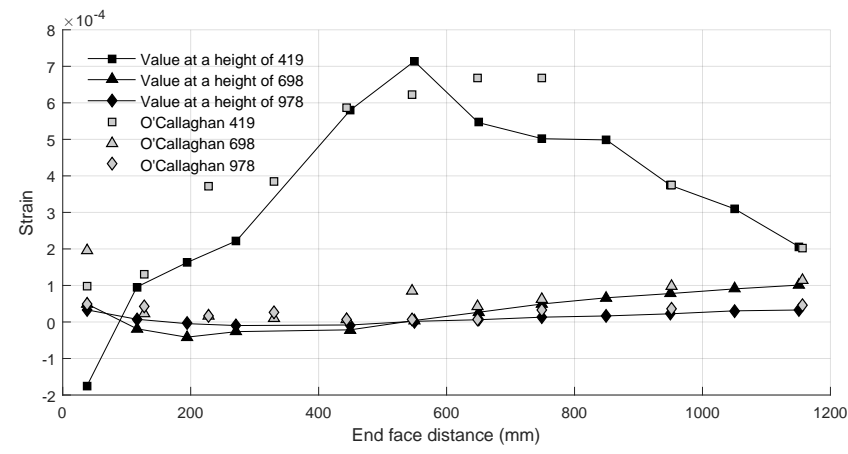

(b) Tx46-girder

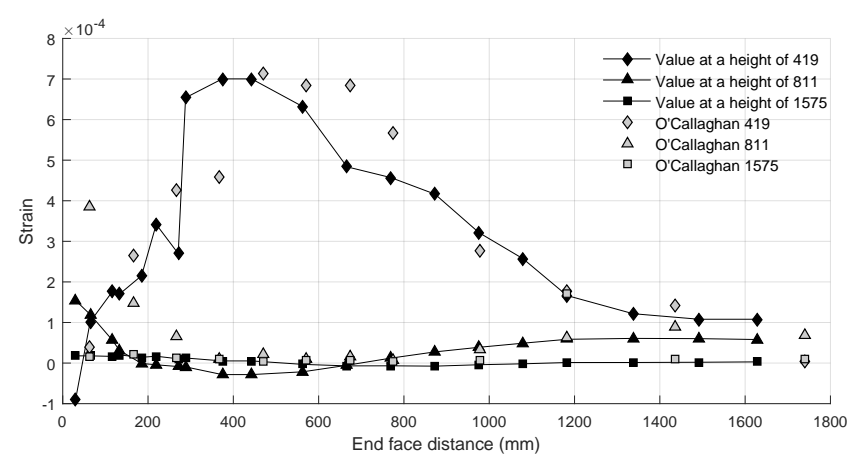

(c) Tx70-girder

Figure 15: Comparison numerical reinforcement strains with experimental results of Tx-girders 